\title{
Midline Prostatic Cysts Presenting with Chronic Prostatitis or Secondary Infertility and Minimally Invasive Treatment: Endoscopic or Laparoscopic Approach?
}

\author{
Hui-hui Zhang, Fan Qi, Jun Wang, Min-feng Chen, Zhuo Li, Xiong-bing Zu \\ Department of Urology, Xiangya Hospital, Central South University, Changsha, China \\ E-mail:whzuxb@163.com \\ Received February 26, 2011; revised May 16, 2011; accepted June 10, 2011
}

\begin{abstract}
Backgrounds: Surgical interventions especially minimally invasive treatments are recommended for symptomatic midline prostatic cysts. The endoscopic unroofing of cysts close to urethra is easy and simple, but it has little effect on the large cysts and cysts lying deeply, in contrast with the laparoscopic approach. Therefore, the selection of minimally invasive therapeutic approaches is important. The aim of this study is to describe our experience in the diagnosis and selection of minimally invasive treatment for midline prostatic cyst. Methods: 15 cases of midline prostatic cyst were studied.10 cases presented with prostatitis-like symptoms, 1 with dysuria and acute urinary retention, 3 with secondary infertility and the rest 1 with hemospermia. 6 patients presented with small cysts $(\leq 2 \mathrm{~cm} \times 2 \mathrm{~cm})$ close to urethra and underwent transurethral unroofing. The other 9 patients with large cysts $(>2 \mathrm{~cm} \times 2 \mathrm{~cm})$ or cysts lying closely behind the prostate received the laparoscopic excision. Results: The average duration of transurethral unroofing and laparoscopic excision was 39 mins and 118 mins respectively, whereas the average time of hospitalization was 2.7 days and 4.5 days respectively. After a follow-up of 21 months, all cases were treated successfully without complications and recurrence. Their prostatitis-like symptoms disappeared, and the three patients presented with secondary infertility achieved conception within one year after the operation. Conclusions: A midline prostatic cyst can present with chronic prostatitis-like symptoms and secondary infertility. It can be cured by minimally invasive treatments, but these procedures should be carefully selected according to the size and location of the cyst.
\end{abstract}

Keywords: Midline Prostatic Cyst, Chronic Prostatitis, Secondary Infertility, Minimally Invasive Treatment, Transurethral Cyst Unroofing, Laparoscopy

\section{Introduction}

Midline prostatic cysts are infrequent and mostly detected incidentally during physical examination. Recently, the widespread availability of transrectal ultrasound has led to an increase in diagnosis of prostatic cysts. It has been reported that midline prostatic cysts were observed in $7.6 \%$ of healthy men and $5 \%$ of symptomatic outpatients $[1,2]$. The majority of patients are symptom-free. However, the enlarged prostatic cyst can compress the adjacent tissues, such as posterior urethra or bladder neck. Then, the patient may suffer from lower urinary tract irritative or obstructive symptoms, epididymitis, perineal and/or inguinal pain [2], which is often misdiagnosed as .
Likewise, the enlarged prostatic cyst can compress the seminal vesicles and cause hematospermia, low ejaculate volume or even infertility [3]. Worse still, malignant degeneration can occur [4]. Thus, although midline prostatic cyst is a rare disease, it should never be neglected. When a patient suffers from recurrent lower urinary tract infection, hematospermia or infertility, the possible of a midline prostatic cyst should be regarded, especially when they had received regular treatments.

Surgical intervention is recommended for symptomatic cysts. Various approaches have been described including endoscopic incision or surgical excision by suprapubic, perineal, or midline transvesical approaches [5]. However, the open surgical excisions are technically 
challenging, usually require a prolonged hospital stay and have increased risks of damage to vital structures [6]. Although the endoscopic incision of cysts is easy and simple, the cysts often recur. Therefore, the effective and minimally invasive therapeutic measures are required. Herein, we present our initial experience with the transurethral unroofing of cysts in 6 patients with small cysts $(\leq 2 \mathrm{~cm} \times 2 \mathrm{~cm})$ close to bladder neck or urethra, and the laparoscopic excision of cysts in 9 patients with large cysts $(>2 \mathrm{~cm} \times 2 \mathrm{~cm})$ or cysts lying closely behind the prostate. From our practice, we find that the minimally invasive treatments are simple and effective therapeutic methods to relieve cyst-related symptoms and settle the problem of sterility caused by midline prostatic cyst. However, the selection of minimally invasive treatment should be judged by the size and location of cysts.

\section{Materials and Methods}

\subsection{Patients and Classification}

From June 2004 to August 2009, 15 patients ranging from 15 to 54 years old (average age 35 ) received minimally invasive treatments for midline prostatic cysts in our department. Prostatitis-like symptoms such as urinary frequency, urinary urgency and perineum discomfort occurred in 10 patients who were treated as chronic prostatitis for 4 - 11 months in other hospitals. Dysuria and acute urinary retention were noted in 1 patient (the youngest) with history of cryptorchidism. 3 patients presented with secondary infertility with low ejaculate volume. Hemospermia was presented in the rest 1 patient.

All 15 patients were divided into two groups according to the volume and location of the cyst estimated by preoperative transrectal ultrasound and CT or MRI. 6 patients with small cysts $(\leq 2 \mathrm{~cm} \times 2 \mathrm{~cm})$ close to bladder neck or urethra underwent transurethral unroofing. 9 patients with large cysts $(>2 \mathrm{~cm} \times 2 \mathrm{~cm})$ or cysts lying closely behind the prostate underwent the laparoscopic excision (Figures 1 and 2).

\subsection{Technique of Transurethral Unroofing}

The patient was placed in a lithotomy position. Transurethral unroofing of the midline cyst was performed under general anaesthesia. The cyst might be found between the bladder neck and the verumontanum and appeared to be obstructing the bladder outlet partly without lateral prostate lobe hypertrophy under cystoscopy. Firstly, the roof of the cyst should be resected adequately under direct vision through resectoscope. Meanwhile, sufficient drainage of cystic fluid was guaranteed. Then the orifice was dilated, allowing complete communication between

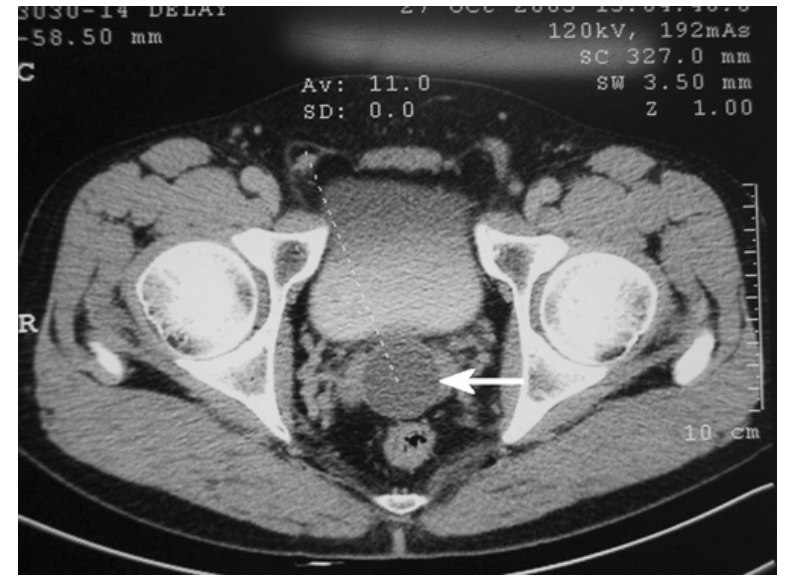

Figure 1. The cyst lies in the midline of prostate (with white arrow).

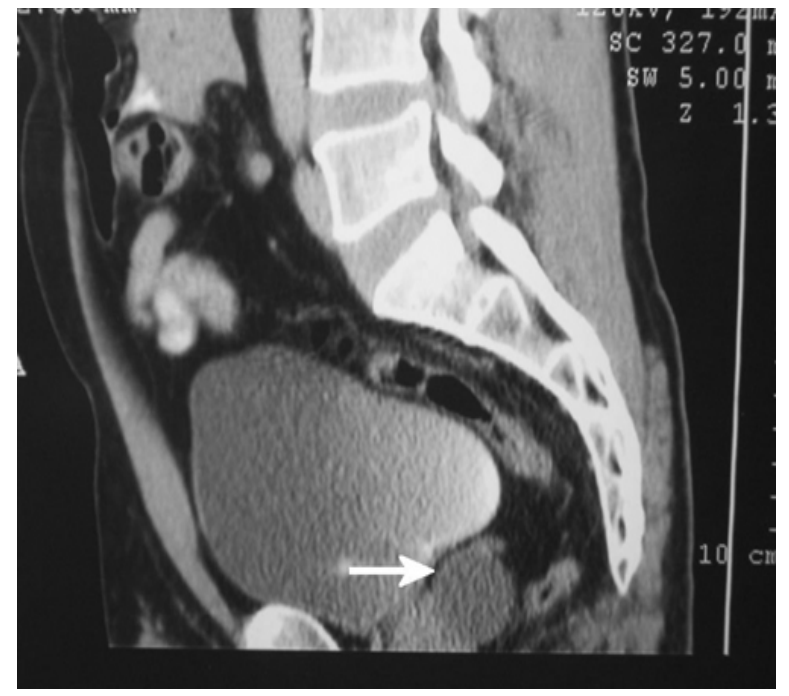

Figure 2. The cyst locates behind the prostate and bladder neck (with white arrow).

the cavity and the uretha. Finally, the lesion was flushed, and the remnant cyst wall was fulgurated circumferentially in order to prevent spontaneous closure. Urinary catheter was indwelled in the bladder and removed $24 \mathrm{~h}$ postoperatively.

\subsection{Technique of Laparoscopic Excision}

The patient was placed in the supine position with the head about $15^{\circ}$ lower with general anesthesia. A $10-\mathrm{mm}$ port was placed on $2-3 \mathrm{~cm}$ below the umbilicus to insert the telescope. Two 5-mm diameter ports were inserted under direct vision, one in each flank to act as operating instrument ports and an additional 3-mm diameter port in the right iliac fossa to act as a bladder retractor [7]. The space of rectovesical excavation might be enlarged by emptying the bladder. The posterior peritoneum covering 
the prostate was then incised, and the cyst could be detected according to exogenous shape and/or preoperative imaging. The prostatic cyst was carefully dissected from adjacent structures. Once dissected completely, the neck of the cyst was ligated with Hem-o-Lok clip and then excised. The cyst wall should be completely excised from the prostate with great attention to avoid recurrence. Finally, the cyst was taken out via the umbilical port.

\section{Results}

There was no prolonged discomfort after both procedures which were well tolerated by all patients. The average duration of transurethral cyst unroofing was 39 mins and the average time of hospitalization was 2.7 days. The average duration of laparoscopic cyst excision was 118 mins and the average time of hospitalization was 4.5 days. All 15 patients showed significant relief of symptoms with neither early nor late postoperative complications, during an average follow-up of 21 months. Semen analysis was performed every 1 - 3 months after surgery for the 3 patient who had presented with secondary infertility. Data revealed that most parameters returned to normal. Their wives conceived within one year after the treatment.

\section{Discussion}

In this study, midline prostatic cysts were defined as hypoechoic to anechoic cystic lesions located in the midline of the prostate detected by transrectal ultrasound [1]. Computed tomography revealed a watery density zone. In most case, the cyst in the midline of the prostate is a mullerian duct cyst or a prostatic utricle cyst. The former originates from the remnants of the mullerian duct, and the latter from the dilatation of the prostatic utricle [8]. Mullerian duct cysts and prostatic utricle cysts may cause obstructive urinary symptoms, irritative symptoms, pelvic pain, epididymal pain, hematuria or infertility. Although it is difficult to distinguish them from each another by imaging and clinical studies, there are still some distinct clinical differences between the two entities. Prostatic utricle cysts tend to arise in younger patients with other urogenital abnormalities, allowing diagnosis at an early stage [9]. Communication with the prostatic urethra is common and sperm may be present in a prostatic utricle cyst. On the contrary, mullerian duct cysts do not communicate with the prostatic urethra and are often discovered later in adults with normal genitalia. Sperm is never found in the mullerian duct cyst [10]. Besides, a prostatic utricle cyst is usually restricted to the prostate region, while the mullerian duct cyst may extend over the base of the prostate and form a visible protru- sion into the bladder [11]. It should be noticed that not all cystic lesions located at the midline of the prostate are müllerian duct cysts or prostatic utricle cysts. The possibility of other cystic lesions should also be considered [12], such as ejaculatory duct cysts, prostatic retention cysts and so on, which are rare in clinical practice. Even so, sometimes conventional terminology indicating each midline prostatic cyst is not adequate to differentiate their subtle variations. Recently, Furuya et al. [13] proposed a new classification of midline prostatic cyst: 1) type 1 , cyst with no communication into the urethra (traditional prostatic utricle cyst); 2) type $2 \mathrm{a}$, cyst with communication into the urethra (cystic dilatation of the prostatic utricle, CDU); 3) type $2 \mathrm{~b}, \mathrm{CDU}$ which communicated with the seminal tract; 4) type 3 (three cases), cystic dilation of the ejaculatory duct. These may help to classify various kinds of midline cysts of the prostate. In practice, however, we always do not further definite characteristics of the midline prostatic cysts because the distinction among these cysts is considered not necessary, due to the same symptoms, anatomical location and primary treatment [2].

Not all the patients with midline prostatic cysts show symptoms. Some cystic lesions in males may be discovered by chance with sonography that is now widely used. In other cases, however, they may remain overlooked. The midline prostatic cyst may lead to various symptoms as described before. Many of these patients are previously diagnosed as prostatitis or urinary tract infection. Although midline prostatic cyst is not a common disease, this entity should be taken into account in the differential diagnosis. For those patients treated as prostatitis or urinary tract infection, which obtained unsatisfactory therapeutic effect repeatly, digital rectal examination is needed. If necessary, sonography, CT or MRI should be carried out additionally.

Treatment is optional for symptomatic cysts and has always been a troublesome problem, when the prostatic cyst lies deep inside the pelvis. Several open surgical ways have been advocated to access the retrourethral space and to remove the cyst by suprapubic, perineal, or midline transvesical approaches as described previously. However, these procedures are technically difficult with a high risk of injury to the adjacent tissues. Furthermore, they may prolong the hospital stay. Therefore, the simple, effective and minimally invasive therapeutic measures are required.

Minimally invasive treatment of the midline prostatic cyst mainly includes transurethral endoscopic approach and laparoscopic approach. Reddy and Winter firstly proposed an endoscopic treatment of the cyst [14]. Although the endoscopic incision of cysts is easy and simple, the cysts often recur. According to our experience, 
transurethral unroofing is well suitable for small prostatic cysts $(\leq 2 \mathrm{~cm} \times 2 \mathrm{~cm})$ close to bladder cavity or urethra. This procedure is also technically simple, with lower risk of complications and shorter hospital stay than open surgical procedures. Moreover, it differs from the endoscopic incision because it causes a thorough drainage, avoiding recurrence. Despite these, disadvantages still exist, because this procedure may forms a wide connective passage from the cyst to the urethra, bringing the risk of retrograde ejaculation into the open cyst cavity and postvoid dribbling. Even worse, It may lead to the infertility subsequently [15]. We noticed that Cornel, et al. [16] had reported only $46 \%$ patients demonstrated an improvement in seminal volume and in one patient improvement of sperm concentration was seen, after transurethral unfoofing of midline prostatic cyst for subfertile men. They listed two reasons to explain the poor efficiency. Firstly, vasography and vesicography were not used in the diagnosis process, a function relationship between the midline prostatic cyst and obstruction was not established. Secondly, the cyst walls were not resected. The edges may heal together once again thus allowing the obstruction to return. From our point of view, an improved patient selection might also influence the result. Patients with large cysts or cysts lying deep often receive unsatisfactory effect after transurethral unfoofing procedure, because it's difficult to accomplish thorough unroofing. Thus, we suggest transurethral unfoofing should only be done in small cysts close to bladder cavity or urethra, ensuring that the cysts can be unroofed thoroughly as to be adequately open to urethra cavity. McDougall, et al. [17] reported the first case of laparoscopic treatment of prostatic cyst. Although this procedure is a litter more difficult than transurethral unroofing and need more time during the operation, it allows a thorough excision of prostatic cysts effectively obviating the disadvantages of transurethral procedure such as postoperative recurrence, retrograde ejaculation and postvoid dribbling. Laparoscopic approach could accomplish complete removal of the cyst in a deep and narrow pelvic cavity with minimal trauma to the normal structures, because it presents a good surgical view with an excellent exposure of all surrounding structures, due to the magnification of surgical field. So this procedure is well suitable for the resection of large midline prostatic cysts and cysts lying deep in the pelvis. The common advantages of laparoscopic approach also include minor incision, less postoperative pain, and earlier return to full recovery [18].

In our present study, we designed a criterion that classified midline prostatic cysts into two groups according to the size and location of cysts through the transrectal ultrasound and CT or MRI. Each group received either the transurethral unroofing or laparoscopic excision of the cyst based on the criterion. Finally, 6 patients with small cysts $(\leq 2 \mathrm{~cm} \times 2 \mathrm{~cm})$ close to bladder cavity or urethra underwent transurethral unroofing. 9 patients with large cysts $(>2 \mathrm{~cm} \times 2 \mathrm{~cm})$ or cysts lying closely behind the prostate received the laparoscopic excision. With the removal of cysts, the symptoms of the present patients were significantly relieved. No complications were recorded. It's worth mentioning that the three patients presented with secondary infertility with low ejaculate volume had remarkable improvement in the ejaculate volume. In addition, in all of them, conception was achieved within one year after the operation.

In conclusion, a midline prostatic cyst can cause chronic prostatitis-like symptoms, and secondary infertility, which is easily neglected. In patients with these symptoms, the prostatic midline cyst must be taken into account for the differential diagnosis. Treatment is necessary for symptomatic patients. Our preliminary results in 15 patients with midline prostatic cysts after minimally invasive treatment showed encouraging therapeutic effects. We suggest that minimally invasive treatments are simple, safe and effective procedures for midline prostatic cysts, but should be selected according to the size and location. Only if we have a correct selection, a convenient operation and good therapeutic effect would be guaranteed. Despite this, our experience is limited by insufficient cases. In the future, more cases and longer follow-up period are required to confirm our experience.

\section{References}

[1] M. Ishikawa, H.Okabe and T. Oya, "Midline Prostatic Cyst in Healthy Men: Incidence and Transabdominal Sonographic Findings," American Journal of Roentgenology, Vol. 181, No. 6, 2003, pp. 1669-1672.

[2] P. Dik, T. M. Lock and B. P. Schrier, "Transurethral Marsupialization of a Medial Prostatic Cyst in Patients with Prostatitis-Like Symptoms," Journal of Urology, Vol. 155, No. 4, 1996, pp. 1301-1304. doi:10.1016/S0022-5347(01)66251-7

[3] J. S. Mayersak, "Urogenital Sinus-Ejaculatory Duct Cyst: a Case Report with a Proposed Clinical Classification and Review of the Literature," Journal of Urology, Vol. 142, No. 5, 1989, pp. 1330-1332.

[4] S. W. Warmann, M. Vogel and M. Wehrmann, "Giant Mullerian Duct Cyst with Malignant Transformation in 15-Year-Old Boy," Urology, Vol. 67, No. 2, 2006, pp. 424-426. doi:10.1016/j.urology.2005.09.009

[5] L. Coppens, P. Bonnet and R. Andrianne, "Adult Mullerian Duct or Utricle Cyst:clinical Significance and Therapeutic Management of 65 Cases," Journal of Urology, Vol. 167, No. 4, 2002, pp. 1740-1744. doi:10.1016/S0022-5347(05)65190-7

[6] C. K. Yeung, J. D. Sihoe and Y. H. Tam, "Laparoscopic Excision of Prostatic Utricles in Children," Brithish Jour- 
nal of Urology International, Vol. 87, No. 6, 2001, pp. 505-508.

[7] I. E. Willetts, J. P. Roberts and A. E. MacKinnon, "Laparoscopic Excision of a Prostatic Utricle in a Child," Pediatric Surgery International, Vol. 19, No. 7, 2003, pp. 557-558. doi:10.1007/s00383-003-0993-6

[8] S. Curran, O. Akin and A. M. Agildere, "Endorectal MRI of Prostatic and Periprostatic Cystic Lesions and Their Mimics," American Journal of Roentgenology, Vol.188, No. 5, 2007, pp. 1373-1379. doi:10.2214/AJR.06.0759

[9] T. D. Schuhrke and G. W. Kaplan, "Prostatic Utricle Cysts (Mullerian Duct Cysts)," Journal of Urology, Vol. 119, No. 6, 1978, pp. 765-767.

[10] S. G. Chang, I. C. Hwang and J. H. Lee, "Infravesical Obstruction due to Benign Intraurethral Prostatic Cyst," Journal of Korean Medical Science, Vol. 119, No. 6, 2003, pp. 125-126.

[11] M. Barzilai and Y. Ginesin, "A Mullerian Prostatic Cyst Protruding into the Base of the Urinary Bladder," Urologia Internationalis, Vol. 60, No. 3, 1998, pp. 194-196. doi:10.1159/000030251

[12] R. Yasumoto, M. Kawano and T. Tsujino, "Is a Cystic Lesion in the Midline of the Prostate a Mullerian Duct Cyst?" European Urology, Vol. 31, No. 2, 1997, pp. 187189.
[13] R. Furuya, S. Furuya and H. Kato, "New Classification of Midline Cysts of the Prostate in Adults via a Transrectal Ultrasonography-Guided Opacification and Dye-Injection Study," Brithish Journal of Urology International, Vol. 102, No. 4, 2008, pp. 475-478.

[14] Y. N. Reddy and C. C. Winter, "Cyst of the Seminal Vesicle: A Case Report and Review of the Literature," Journal of Urology, Vol. 108, No. 1, 1972, pp. 134-135.

[15] R. Anding, F. Steinbach and T. M. Bernhardt, "Urology Treatment of Large Prostatic Cyst with Retropubic Insertion of a Fat Tissue Flap," Journal of Urology, Vol. 164, No. 2, 2000, pp. 454-455. doi:10.1016/S0022-5347(05)67390-9

[16] E. B. Cornel, G. R. Dohle and E. J. Meuleman, "Transurethral Deroofing of Midline Prostatic Cyst for Subfertile Men," Human Reproduction, Vol. 14, No. 9, 1999, pp. 2297-2300. doi:10.1093/humrep/14.9.2297

[17] E. M. McDougall, R. V. Clayman and W. T. Bowles, "Laparoscopic Excision of Müllerian Duct Remnant," Journal of Urology, Vol. 152, No. 2, 1972, pp. 482-484.

[18] J. H. Luo, W. Chen and J. J. Sun, "Laparoscopic Management of Müllerian Duct Remnants: 4 Cases Report and Review of the Literature," Journal of Andrology, Vol. 29, No. 6, 2008, pp. 638-642. doi:10.2164/jandrol.108.005496 